\title{
Segmental Enteritis Associated with Pythium insidiosum Infection in a Horse
}

\author{
Taiara Müller da Silva', Mariana Martins Flores ${ }^{3}$, Eryca Ceolin Lamego', Douglas Miotto Lorenzetti', \\ Cristina Prade Ramos² \& Glaucia Denise Kommers ${ }^{3}$
}

\begin{abstract}
Background: Pythiosis is an infectious disease caused by the oomycete Pythium insidiosum, with higher occurrence in wetlands and hot climate regions. This microorganism develops its cycle in aquatic plants, and most cases happen because of the contact of animals or people with water containing the motile zoospores (infectious form). Horses are the principal species affected and develop principally cutaneous and subcutaneous lesions, but the gastrointestinal tract is seldom affected. Humans develop various forms of pythiosis, such as a vascular form. The objectives of the current study are to describe an unusual case of intestinal pythiosis, its clinical signs, aspects of pathogenesis, and diagnosis.

Case: A 13-year-old Crioula mare, from Santa Maria, RS, Brazil, presented with reduced food and water intake, apathy, restlessness, rolling, nasal reflux, firm abdomen upon palpation, and tachypnea during 2 days. The horses of this farm were fed native pasture and horse feed, and they had access to a nearby pond. Two days following the start of the clinical signs, the horse died and was necropsied on the farm. During necropsy, there was around $400 \mathrm{~mL}$ of reddish effusion in the abdominal cavity (modified transudate). A $15 \mathrm{~cm}$ segment of jejunum was firm upon palpation and had a severe transmural thickening. The wall of the affected area was up to $3 \mathrm{~cm}$ in thickness and firm, with small yellowish and irregular masses that stood out and looked friable, interpreted as kunkers. Microscopically, the yellowish masses (kunkers) were characterized by dense accumulations of intact and degenerate eosinophils (eosinophilic necrosis). Within these kunkers, and also on their periphery, there were multiple negatively stained hyphal profiles. Hyphae were also seen on the wall of small arteries inside the kunkers. These hyphae had nearly parallel walls and were occasionally septate, with a diameter of about $10 \mu \mathrm{m}$. The hyphae stained black with Grocott's methenamine silver stain (GMS) and were positive on immunohistochemistry (IHC) using specific anti-P. insidiosum polyclonal antibody.

Discussion: Colic is the principal clinical presentation in horses suffering from gastrointestinal tract disorders. In spite of the presence of colic in this horse, intestinal pythiosis was not clinically suspected in this case, principally because of its rare occurrence in horses. Even though it is persistent to observe horses ingesting water with suspected contamination by $P$. insidiosum, the cutaneous form of pythiosis is a lot more common than the intestinal form. This is the only case of intestinal pythiosis in a horse in 52 years of routine diagnosis in our laboratory. It is suspected that this horse got infected by drinking contaminated water from the nearby pond and microlesions in the intestinal mucosa due to plant material or some unknown pathogen may have favored zoospore adhesion, encysting and starting the colonization of the tissue by emitting a germ tube. A macroscopic observation of kunkers in the intestine is rare, but when observed it is highly indicative of pythiosis. The immunohistochemistry technique using anti-P. insidiosum antibody, in accordance with the literature, validates the diagnosis of pythiosis. In this case, the presence of hyphae in the blood vessel wall inside the kunkers propose that this finding may be involved in the spread of the lesion but needs more detailed studies.
\end{abstract}

Keywords: intestinal pythiosis, horse, oomycete, hyphae, immunohistochemistry. 


\section{INTRODUCTION}

Pythiosis is an infectious disease due to the oomycete Pythium insidiosum, with greater occurrence in wetlands as well as hot climate regions. This microorganism develops its cycle in aquatic plants, and most cases happen because of the contact of animals or people with water containing the motile zoospores (infective form) [7]. Horses are the principal species affected and develop principally cutaneous and subcutaneous lesions, because of their natural habit wherein they stay for long periods in ponds or lakes. Despite this, the gastrointestinal tract is seldom affected in horses, which is different in what is seen in the canine species [12]. Probably, the lesion development occurs by contact of motile zoospores with skin or mucous membranes that have prior microlesions [7]. Humans develop different forms of pythiosis, such as a vascular form [10]. The aims of the current study are to describe an unusual case of intestinal pythiosis, including clinical signs, main lesions, aspects of pathogenesis, and diagnosis.

\section{CASE}

A 13-year-old Crioula mare, from Santa Maria, RS, Brazil, presented with a reduced food and water intake, apathy, restlessness, rolling, nasal reflux of bloody fluid, firm abdomen upon palpation, and tachypnea. Two days following the beginning of the clinical signs, the horse died and was necropsied on the farm. The horses of this farm were fed with native pasture and horse feed and had access to a nearby pond.

During necropsy, there was around $400 \mathrm{~mL}$ of reddish effusion in the abdominal cavity (modified transudate). A $15 \mathrm{~cm}$ segment of the jejunum was firm upon palpation and had a severe transmural thickening. The wall of the affected area was up to $3 \mathrm{~cm}$ in thickness and firm, with small yellowish and irregular masses that stood out and looked friable, which are interpreted as kunkers (Figure 1). The mucosa was also ulcerated with a darkened luminal surface. Adhesion of the mesentery to the serosa of the affected segment was noted. Prior to the affected area, the duodenal and jejunal segments demonstrated wall edema and multifocal petechiae on serosa. The entire small intestine was filled with bloody fluid content.

Tissue samples were formalin-fixed and routinely processed for histopathology in the Laboratório de Patologia Veterinária of the Universidade Federal de Santa Maria (LPV-UFSM). Microscopically, the yellowish masses (kunkers) were distributed multifocally within the wall of the affected intestinal segment and were characterized by dense accumulations of intact as well as degenerate eosinophils (eosinophilic necrosis) [Figure 2]. Within these kunkers, and also on their periphery, there were multiple negatively stained hyphal profiles. These hyphae had nearly parallel walls and were occasionally septate, with a diameter of around $10 \mu \mathrm{m}$. The hyphae stained black with Grocott's methenamine silver stain - GMS ${ }^{1}$ (histokit Grocott - EP-12-20017) [Figure 3]. Hyphae were also noted on the wall of small degenerate arteries inside of some kunkers (Figure 4). To confirm the etiology, IHC was conducted using an anti-P. insidiosum polyclonal antibody ${ }^{1}$, according to Gabriel et al. [4], modified by Martins et al. [6]. The hyphae were positive on IHC (Figure 5). In 52 years (1968-2019), 609 horses have been necropsied in the LPV-UFSM, and this is the only case of intestinal pythiosis.

\section{DISCUSSION}

The gross and histological findings of this case were highly consistent with intestinal pythiosis. The use of hematoxylin and eosin and GMS does not distinguish between Pythium insidiosum, Conidiobolus spp., and Basidiobolus spp. hyphae [11]. Therefore, as in this case, the confirmation of the etiology can be made by IHC using anti-P. insidiosum specific antibody, which has been utilized reliably since 1988 [3] and is well established by different authors [4-6,11].

The mural thickening of the jejunum of this horse developed severe clinical signs of colic. This clinical syndrome is the principal clinical presentation in horses with gastrointestinal tract disorders [1,9]. This is the first case of intestinal pythiosis in a horse at LPV-UFSM in 52 years, making up $0.16 \%$ of the cases. Therefore, in spite of the presence of colic in this horse, intestinal pythiosis was not clinically suspected in this case. This fact shows that intestinal pythiosis should be included in the list of differential diagnoses of colic.

Intestinal masses in live horses must be clinically differentiated from tumors, foreign bodies, enteroliths, phytobezoars, impactions, intussusceptions, and severe intestinal inflammation. When macroscopic evaluation excludes foreign bodies and enteroliths, circumferential masses in the intestinal wall should be distinguished from neoplasms (lymphoma, adenocarcinoma) and severe intestinal inflammation. Histopatho- 


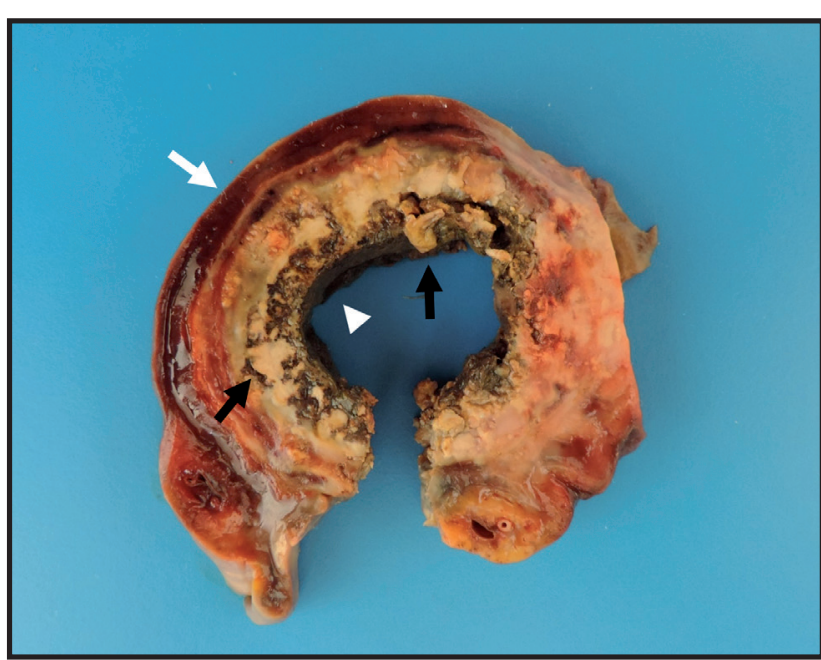

Figure 1. Cutting surface with thickening of the wall and multiple yellowish masses near the mucosa (black arrows) and wall edema (white arrow). Ulceration of the intestinal mucosa (arrow head) is observed.

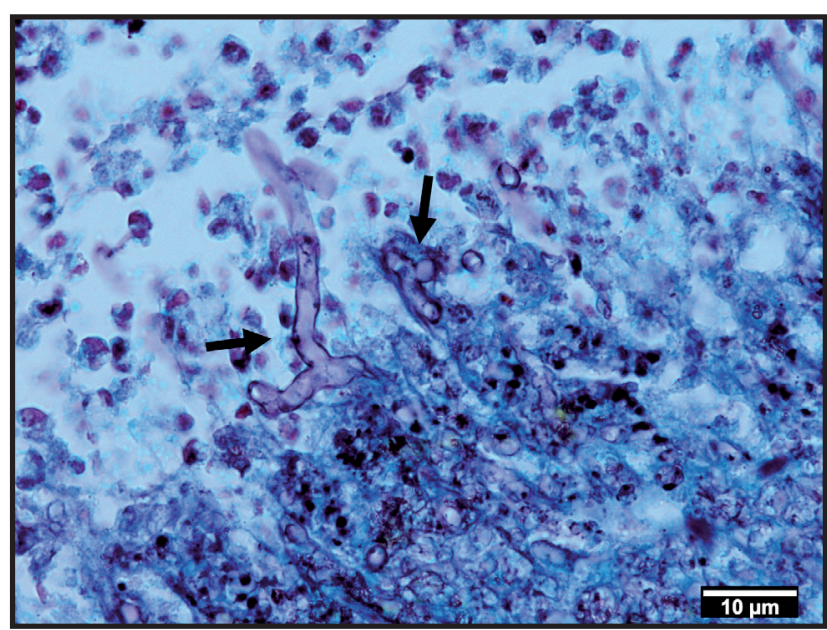

Figure 3. Hyphae impregnated in black by silver (arrows) are noted in the peripheral region of a kunker [GMS; bar= $10 \mu \mathrm{m}$ ].

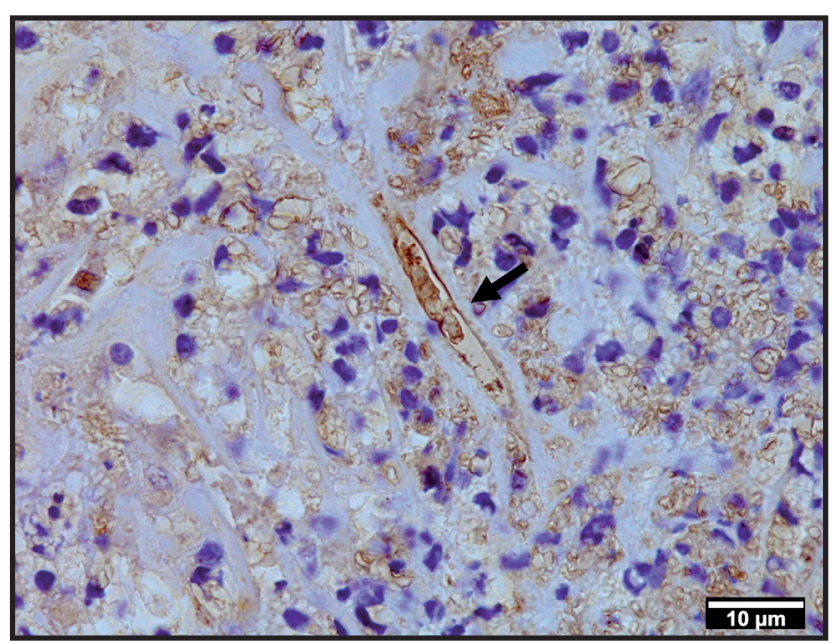

Figure 5. A hyphae immunolabelled (arrow) for polyclonal anti-Pythium insidiosum antibody by immunohistochemical staining [IHC; bar $=10 \mu \mathrm{m}]$.

logical analysis of biopsied masses affecting the intestines can help in the diagnosis of intestinal pythiosis in live animals. In the case of pythiosis, there may be, in

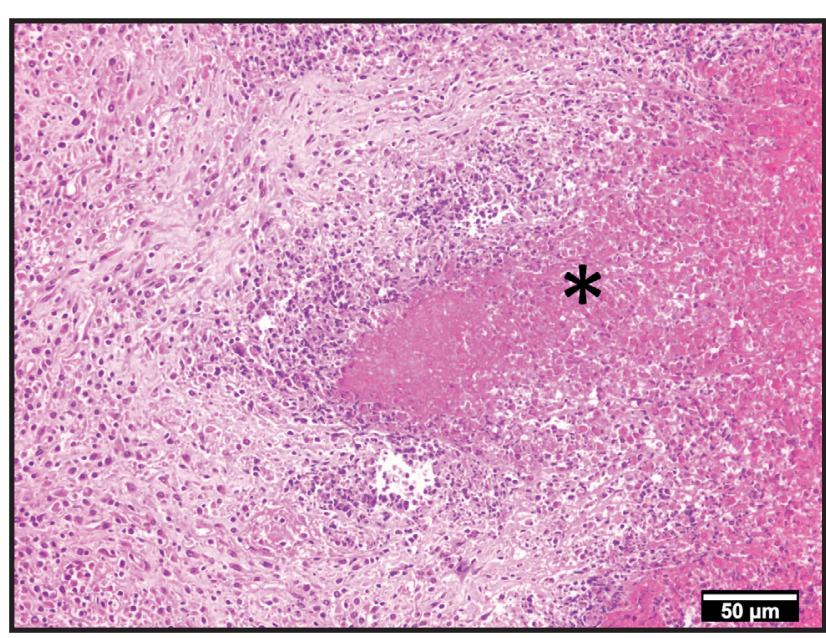

Figure 2. Approximate image of a kunker (asterisk) showing a dense focus with large numbers of intact and degenerate eosinophils with eosinophil granules (eosinophilic necrosis). Adjacent to the kunker, an abundant granulation tissue was seen [hematoxylin and eosin; bar $=50 \mu \mathrm{m}$ ].

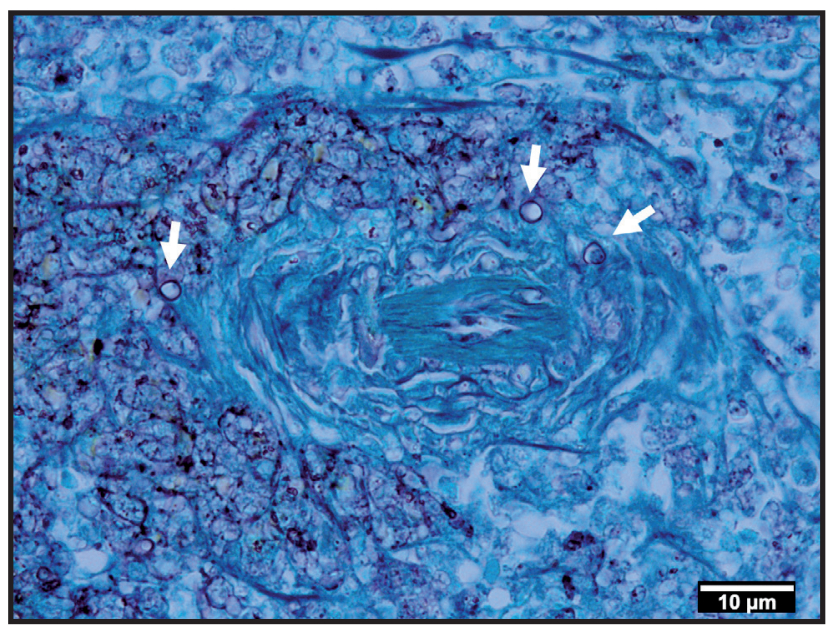

Figure 4. Hyphae impregnated in black (arrows) by silver in the artery wall inside kunkers [GMS; bar $=10 \mu \mathrm{m}]$.

the middle of these masses, small concretions called kunkers [12], as seen in this case. Cases wherein the animal was submitted to surgery and recovered from intestinal pythiosis have also been reported $[1,8]$. But invariably, in some cases, the injury is so extensive that the animal must be submitted to euthanasia [9].

The cutaneous form of pythiosis is a lot more common than the intestinal form. Although it is frequent to observe horses drinking suspected contaminated water by $P$. insidiosum, there are a few reports of the latter in the literature $[1-3,8,9]$. It is suspected that this horse got infected by drinking contaminated water from the nearby pond; however, a microbiological evaluation of the water was not performed to validate this hypothesis.

The pathogenesis of intestinal pythiosis lesions is not well understood [2]. However, it is believed that microlesions on the intestinal mucosa due to plant ma- 
terial or pathogenic agents may favor zoospore adhesion, encystation, and initiation of tissue colonization via the emission of a germ tube [7]. In this case, it was not possible to confirm the pre-existence of microlesions, because the intestinal lesions observed during necropsy were advanced and would have concealed possible pre-existent abrasions or ulcers. The formation of kunkers in equine pythiosis is classically seen in skin lesions and seldom reported in the intestines [1]. Inside some kunkers, hyphae were also seen on the wall of small arteries. This finding proposes a possible role of the vascular wall in the spread of pythiosis lesions, as noted in humans, in which vascular lesions facilitate the dissemination of hyphae [10].

Colic due to intestinal pythiosis is an uncommon finding in horses. However, it should be included in the differential diagnosis of this condition. In this case, it was characterized by the presence of segmental enteritis with kunkers, characteristic histopathological findings, and etiology confirmation by immunohistochemistry. The observation of hyphae in the artery wall inside kunkers suggest their involvement in the pathogenesis; however, it needs further detailed studies.

\section{MANUFACTURER}

${ }^{1}$ EasyPath. Indaiatuba, SP, Brazil.

Acknowledgments. The authors are grateful to the Coordenação de Aperfeiçoamento de Pessoal de Nível Superior (CAPES) for the financial support. T. M. Silva has a scholarship from CAPES, and E. C. Lamego had a scholarship from the Fundação de Amparo à Pesquisa do Estado do Rio Grande do Sul (FAPERGS). The authors are grateful to the Laboratório de Pesquisas Micológicas (LAPEMI-UFSM) for the anti-Pythium insidiosum antibody.

Declaration of interest. The authors declare no conflicts of interest. The authors alone are responsible for the content and writing of this paper.

\section{REFERENCES}

1 Allison N. \& Gillis J.P. 1990. Enteric pythiosis in a horse. Journal of the American Veterinary Medical Association. 196: 462-464.

2 Bezerra Júnior P.S., Pedroso P.M.O., Pavarini S.P., Dalto A.G.C., Santurio J.M. \& Driemeier D. 2010. Equine intestinal pythiosis in Southern Brazil. Arquivo Brasileiro de Medicina Veterinária e Zootecnia. 62: 481-483. DOI: 10.1590/S0102-09352010000200031.

3 Brown C.C. \& Roberts E.D. 1988. Intestinal pythiosis in a horse. Australian Veterinary Journal. 65: 88-89.

4 Gabriel A.L., Kommers G.D., Trost M.E., Barros C.S.L., Pereira D.B., Schwendler S.E. \& Santurio J.M. 2008. Surto de pitiose cutânea em bovinos. Pesquisa Veterinária Brasileira. 28: 583-587. DOI: 10.1590/S0100736X2008001200003.

5 Galiza G.J.N., Tochetto C., Rosa F.B., Panziera W., Silva T.M., Caprioli R.A. \& Kommers G.D. 2014. Utilização de três métodos imuno-histoquímicos na detecção de aspergilose e zigomicose em animais. Pesquisa Veterinária Brasileira. 34: 637-642. DOI: 10.1590/S0100-736X2014000700005.

6 Martins T.B., Kommers G.D., Trost M.E., Inkelmann M.A., Fighera R.A. \& Schild A.L. 2012. A Comparative Study of the Histopathology and Immunohistochemistry of Pythiosis in Horses, Dogs and Cattle. Journal Comparative Pathology. 146: 122-131. DOI: 10.1016/j.jcpa.2011.06.006.

7 Mendoza L., Hernandez F. \& Ajello L. 1993. Life Cycle of the Human and Animal Oomycete Pathogen Pythium insidiosum. Journal of Clinical Microbiology. 31: 2967-2973.

8 Morton L.D., Morton D.G., Baker G.J. \& Gelberg H.B. 1991. Chronic Eosinophilic Enteritis Attributed to Pythium sp. in a Horse. Veterinary Pathology. 28: 542-544.

9 Purcell K.L., Johnson P.J., Kreeger J.M. \& Wilson D.A. 1994. Jejunal obstruction caused by a Pythium insidiosum granuloma in a mare. Journal of the American Veterinary Medical Association. 205: 237-239.

10 Reanpang T., Orrapin S., Orrapin S., Arworn S., Kattipatanapong T., Srisuwan T., Vanittanakom N., Lekawanvijit S.P. \& Rerkasem K. 2015. Vascular Pythiosis of the Lower Extremity in Northern Thailand: Ten Years' Experience. The International Journal of Lower Extremity Wounds. 14: 245-250. DOI: 10.1177/1534734615599652.

11 Ubiali D.G, Cruz R.A.S., De Paula D.A.J., Silva M.C., Mendonça F.S., Dutra V., Nakazato L., Colodel E.M. \& Pescador C.A. 2013. Pathology of nasal infection caused by Conidiobolus lamprauges and Pythium insidiosum in sheep. Journal of Comparative Pathology. 149: 137-145. DOI: 10.1016/j.jcpa.2012.12.002.

12 Uzal F.A., Plattner B.L. \& Hostetter J.M. 2016. Alimentary System. In: Maxie M.G. (Ed). Jubb, Kennedy, and Palmer's Pathology of Domestic Animals. 6th edn. St. Louis: Elsevier, pp.1-257. 